\title{
Importance of short-wavelength excitation in environmental vibrations due to urban rail traffic
}

Environmental vibrations induced by urban railway traffic are mainly generated from uneven wheel-rail contact in the short wavelength range, according to a study published in Science China Technological Sciences, 2012, Vol. 55(4).

The introduction of urban railways is one of the most effective ways to deal with traffic problems in large cities. However, in the vicinity of railways, day-by-day vibrations from trains may cause discomfort to people, the malfunctioning of sensitive equipment, and even damage to old buildings, and railways therefore have serious environmental problems. The development of urban railways is now limited by such environmental vibrations.

Effective vibration-reduction technologies rely on a good understanding of the excitation source that generates the vibrations. "In recent years, substantial progress has been made in modeling the train-track-ground interaction, and consensus has been reached that the excitation source is the moving of constant loads and uneven contact between wheels and rails," according to background information in the article. "Nevertheless, the contact can hardly be measured directively, so its amplitude and frequency contents are not completely understood, and its quantitative expression remains a problem for further research to address."

Recently, a research group led by Prof. Tao XiaXin at Harbin Institute of Technology, China, has made a breakthrough in revealing the excitation mechanism. Based on an inversion study in the frequency and wave-number domain, Dr. Wang FuTong, a key research member in the group, has found that high-frequency contents are predominant in the excitation. A power spectral density function (PSD) of uneven wheel-rail contact, rather than the track PSD, was suggested to describe the random characteristics of the excitation source. An inversion strategy was then established to obtain the source function from vibration data recorded by an observation array at the ground surface. The wheel-rail unevenness PSD, being the source function for the No. 13 Beijing urban railway, was obtained by the inversion strategy. The result indicated that the source function properly described the track unevenness in the range of wavelengths over $1.2 \mathrm{~m}$, and showed wheel irregularities in the range of wavelengths shorter than $1.2 \mathrm{~m}$.

The researchers found that, in the range of short wavelengths under $1.2 \mathrm{~m}$, the wheel-rail PSD maintained a value higher than the 6th class of the track PSD suggested by the Federal Railway Administration. As urban trains do not travel particularly quickly, this short wavelength range exactly corresponds to the main frequency band of environmental vibrations; i.e. the frequency components of the vibrations stem mainly from that range of uneven excitation. Taking account of only moving constant loads and track unevenness could result in a severe underestimation of the environmental vibrations.

"Whereas the track spectrum reflects only the evenness of the track, the wheel-rail spectrum expresses both the track unevenness and the irregularities of wheels, and it is therefore more suitable to be used as the source function of urban railway traffic," the researchers write. "It is also shown that inversion of the exciting source according to observed ground vibrations is an effective way to detect quantitatively the combined wheel-rail unevenness."

See the article: Wang F T, Tao X X, Zheng X. Inversion of excitation source in ground vibration from urban railway traffic. Sci China Tech Sci, 2012, 55: 950-959

Open Access This article is distributed under the terms of the Creative Commons Attribution License which permits any use, distribution, and reproduction in any medium, provided the original author(s) and source are credited. 